\title{
ON LINEAR VOLTERRA DIFFERENCE EQUATIONS WITH INFINITE DELAY
}

\author{
CH. G. PHILOS AND I. K. PURNARAS \\ Received 2 February 2005; Revised 30 June 2005; Accepted 6 July 2005
}

Linear neutral, and especially non-neutral, Volterra difference equations with infinite delay are considered and some new results on the behavior of solutions are established. The results are obtained by the use of appropriate positive roots of the corresponding characteristic equation.

Copyright $\odot 2006$ Ch. G. Philos and I. K. Purnaras. This is an open access article distributed under the Creative Commons Attribution License, which permits unrestricted use, distribution, and reproduction in any medium, provided the original work is properly cited.

\section{Preliminary notes}

Motivated by the old but significant papers by Driver [3] and Driver et al. [5], a number of relevant papers has recently appeared in the literature. See Frasson and Verduyn Lunel [10], Graef and Qian [11], Kordonis et al. [16], Kordonis and Philos [19], Kordonis et al. [21], Philos [26], and Philos and Purnaras [28, 30, 35, 33, 36]. The results in [10, 11, $16,26,28,30,35,36]$ concern the large time behavior of the solutions of several classes of linear autonomous or periodic delay or neutral delay differential equations, while those in $[19,21,33]$ are dealing with the behavior of solutions of some linear (neutral or nonneutral) integrodifferential equations with unbounded delay. Note that the method used in [10] is based on resolvent computations and Dunford calculus, while the technique applied in the rest of the papers mentioned above is very simple and is essentially based on elementary calculus. We also notice that the article [10] is very interesting as well as comprehensive.

Along with the work mentioned above for the continuous case, analogous investigations have recently been made for the behavior of the solutions of some classes of linear autonomous or periodic delay or neutral delay difference equations, for the behavior of the solutions of certain linear delay difference equations with continuous variable as well as for the behavior of solutions of a linear Volterra difference equation with infinite delay. See Kordonis and Philos [17], Kordonis et al. [20], and Philos and Purnaras 
$[29,31,32,34]$. For some related results we refer to the papers by de Bruijn [2], Driver et al. [4], Györi [12], Norris [25], and Pituk [37, 38].

In [21], Kordonis et al. obtained some results on the behavior of solutions of linear neutral integrodifferential equations with unbounded delay; the results in [21] extend and improve previous ones given by Kordonis and Philos [19] for the special case of (non-neutral) integrodifferential equations with unbounded delay. In [33], Philos and Purnaras continued the study in $[19,21]$ and established some further results on the behavior of solutions of linear neutral integrodifferential equations with unbounded delay, and, especially, of linear (non-neutral) integrodifferential equations with unbounded delay.

Our purpose in this paper is to give the discrete analogues of the results in $[19,21,33]$. Here, we study the behavior of solutions of linear neutral Volterra difference equations with infinite delay, and, especially, of linear (non-neutral) Volterra difference equations with infinite delay. Our results will be derived by the use of appropriate positive roots of the corresponding characteristic equation. Some of the results of the present paper extend and improve the main results of the authors' previous paper [32].

Neutral, and especially non-neutral, Volterra difference equations with infinite delay have been widely used as mathematical models in mathematical ecology, particularly in population dynamics. Although the bibliography on Volterra integrodifferential equations is quite extended, however there has not yet been analogously much work on the Volterra difference equations. We choose to refer here to the papers by Jaroš and Stavroulakis [13], Kiventidis [15], Kordonis and Philos [18], Ladas et al. [22], and Philos [27] for some results concerning the existence and/or the nonexistence of positive solutions of certain linear Volterra difference equations. Also, for some results on the stability of Volterra difference equations, we typically refer to the papers by Elaydi $[6,8]$, and Elaydi and Murakami [9] (see, also, the book [7, pages 239-250]).

For the general background of difference equations, one can refer to the books by Agarwal [1], Elaydi [7], Kelley and Peterson [14], Lakshmikantham and Trigiante [23], Mickens [24], and Sharkovsky et al. [39].

The paper is organized as follows. Section 2 contains an introduction and some notations. Section 3 is devoted to the statement of the main results (and to some comments on them). The proofs of the main results will be given in Section 4 .

\section{Introduction and notations}

Throughout the paper, $\mathbf{N}$ stands for the set of all nonnegative integers and $\mathbf{Z}$ stands for the set of all integers. Also, the set of all nonpositive integers will be denoted by $\mathbf{Z}^{-}$. Moreover, the forward difference operator $\Delta$ will be considered to be defined as usual, that is,

$$
\Delta s_{n}=s_{n+1}-s_{n}, \quad n \in \mathbf{N}
$$

for any sequence $\left(s_{n}\right)_{n \in \mathrm{N}}$ of real numbers.

Consider the linear neutral Volterra difference equation with infinite delay

$$
\Delta\left(x_{n}+\sum_{j=-\infty}^{n-1} G_{n-j} x_{j}\right)=a x_{n}+\sum_{j=-\infty}^{n-1} K_{n-j} x_{j}
$$


and, especially, the linear (non-neutral) Volterra difference equation with infinite delay

$$
\Delta x_{n}=a x_{n}+\sum_{j=-\infty}^{n-1} K_{n-j} x_{j}
$$

where $a$ is a real number, and $\left(G_{n}\right)_{n \in \mathbf{N}-\{0\}}$ and $\left(K_{n}\right)_{n \in \mathbf{N}-\{0\}}$ are sequences of real numbers. It will be supposed that $\left(K_{n}\right)_{n \in \mathrm{N}-\{0\}}$ is not eventually identically zero. Note that $(2.3)$ is a special case of (2.2), that is, the special case where the kernel $\left(G_{n}\right)_{n \in \mathbf{N}-\{0\}}$ is identically zero.

Equation (2.2) can equivalently be written as follows

$$
\Delta\left(x_{n}+\sum_{j=1}^{\infty} G_{j} x_{n-j}\right)=a x_{n}+\sum_{j=1}^{\infty} K_{j} x_{n-j}
$$

and, especially, (2.3) can equivalently be written as

$$
\Delta x_{n}=a x_{n}+\sum_{j=1}^{\infty} K_{j} x_{n-j}
$$

By a solution of the neutral Volterra difference equation (2.2) (respectively, of the (nonneutral) Volterra difference equation (2.3)), we mean a sequence $\left(x_{n}\right)_{n \in \mathbf{Z}}$ of real numbers which satisfies (2.2) (resp., (2.3)) for all $n \in \mathbf{N}$.

In the sequel, by $S$ we will denote the (nonempty) set of all sequences $\phi=\left(\phi_{n}\right)_{n \in \mathbf{Z}^{-}}$of real numbers such that, for each $n \in \mathbf{N}$,

$$
\Phi_{n}^{G} \equiv \sum_{j=-\infty}^{-1} G_{n-j} \phi_{j}=\sum_{j=n+1}^{\infty} G_{j} \phi_{n-j}, \quad \Phi_{n}^{K} \equiv \sum_{j=-\infty}^{-1} K_{n-j} \phi_{j}=\sum_{j=n+1}^{\infty} K_{j} \phi_{n-j}
$$

exist in R. In the special case of (2.3), the set $S$ consists of all sequences $\phi=\left(\phi_{n}\right)_{n \in \mathbf{Z}^{-}}$of real numbers such that, for each $n \in \mathbf{N}, \Phi_{n}^{K}$ exists in $\mathbf{R}$.

It is clear that, for any given initial sequence $\phi=\left(\phi_{n}\right)_{n \in \mathbf{Z}^{-}}$in $S$, there exists a unique solution $\left(x_{n}\right)_{n \in \mathbf{Z}}$ of the difference equation (2.2) (resp., of (2.3)) which satisfies the initial condition

$$
x_{n}=\phi_{n} \quad \text { for } n \in \mathbf{Z}^{-}
$$

this solution $\left(x_{n}\right)_{n \in \mathrm{Z}}$ is said to be the solution of the initial problem (2.2) and (2.7) (resp., of the initial problem (2.3) and (2.7)) or, more briefly, the solution of (2.2) and (2.7) (resp., of (2.3) and (2.7)).

With the neutral Volterra difference equation (2.2) we associate its characteristic equation

$$
(\lambda-1)\left(1+\sum_{j=1}^{\infty} \lambda^{-j} G_{j}\right)=a+\sum_{j=1}^{\infty} \lambda^{-j} K_{j}
$$


which is obtained by seeking solutions of (2.2) of the form $x_{n}=\lambda^{n}$ for $n \in \mathbf{Z}$, where $\lambda$ is a positive real number. In particular, the characteristic equation of the (non-neutral) Volterra difference equation (2.3) is

$$
\lambda-1=a+\sum_{j=1}^{\infty} \lambda^{-j} K_{j} .
$$

The use of a positive root $\lambda_{0}$ of the characteristic equation (2.8) with the property

$$
\sum_{j=1}^{\infty} \lambda_{0}^{-j}\left(1+\left|1-\frac{1}{\lambda_{0}}\right| j\right)\left|G_{j}\right|+\frac{1}{\lambda_{0}} \sum_{j=1}^{\infty} \lambda_{0}^{-j} j\left|K_{j}\right|<1
$$

plays a crucial role in obtaining the results of this paper. In the special case of the (nonneutral) Volterra difference equation (2.3), the property (2.10) (of a positive root $\lambda_{0}$ of the characteristic equation (2.9)) takes the form

$$
\frac{1}{\lambda_{0}} \sum_{j=1}^{\infty} \lambda_{0}^{-j} j\left|K_{j}\right|<1
$$

In what follows, if $\lambda_{0}$ is a positive root of (2.8) (resp., of (2.9)) with the property (2.10) (resp., with the property (2.11)), we will denote by $S\left(\lambda_{0}\right)$ the (nonempty) subset of $S$ consisting of all sequences $\phi=\left(\phi_{n}\right)_{n \in \mathbf{Z}^{-}}$in $S$ such that $\left(\lambda_{0}^{-n} \phi_{n}\right)_{n \in \mathbf{Z}^{-}}$is a bounded sequence.

Now, we introduce certain notations which will be used throughout the paper without any further mention. We also give some facts concerning these notations that we will keep in mind in what follows.

Let $\lambda_{0}$ be a positive root of the characteristic equation (2.8) with the property (2.10). We define

$$
\begin{aligned}
& \gamma\left(\lambda_{0}\right)=\sum_{j=1}^{\infty} \lambda_{0}^{-j}\left[1-\left(1-\frac{1}{\lambda_{0}}\right) j\right] G_{j}+\frac{1}{\lambda_{0}} \sum_{j=1}^{\infty} \lambda_{0}^{-j} j K_{j}, \\
& \mu\left(\lambda_{0}\right)=\sum_{j=1}^{\infty} \lambda_{0}^{-j}\left(1+\left|1-\frac{1}{\lambda_{0}}\right| j\right)\left|G_{j}\right|+\frac{1}{\lambda_{0}} \sum_{j=1}^{\infty} \lambda_{0}^{-j} j\left|K_{j}\right| .
\end{aligned}
$$

Property (2.10) together with the hypothesis that $\left(K_{n}\right)_{n \in \mathbf{N}-\{0\}}$ is not eventually identically zero guarantee that

$$
0<\mu\left(\lambda_{0}\right)<1
$$

Also, because of $\left|\gamma\left(\lambda_{0}\right)\right| \leq \mu\left(\lambda_{0}\right)$, we have $-1<\gamma\left(\lambda_{0}\right)<1$, that is,

$$
0<1+\gamma\left(\lambda_{0}\right)<2
$$

In the particular case where $\left(G_{n}\right)_{n \in \mathbf{N}-\{0\}}$ and $\left(K_{n}\right)_{n \in \mathbf{N}-\{0\}}$ are nonpositive and $\lambda_{0}$ is less than or equal to 1 , because of the fact that $\left(K_{n}\right)_{n \in \mathrm{N}-\{0\}}$ is not eventually identically zero, the property (2.10) can be written as $-1<\gamma\left(\lambda_{0}\right)<0$, that is,

$$
0<1+\gamma\left(\lambda_{0}\right)<1
$$


Furthermore, we set

$$
\Theta\left(\lambda_{0}\right)=\frac{\left[1+\mu\left(\lambda_{0}\right)\right]^{2}}{1+\gamma\left(\lambda_{0}\right)}+\mu\left(\lambda_{0}\right) .
$$

We can easily see that $\Theta\left(\lambda_{0}\right)$ is a real number with

$$
\Theta\left(\lambda_{0}\right)>1
$$

Let us consider the special case of the (non-neutral) Volterra difference equation (2.3) and let $\lambda_{0}$ be a positive root of the characteristic equation (2.9) with the property (2.11). In this case, we define

$$
\begin{aligned}
& \gamma_{0}\left(\lambda_{0}\right)=\frac{1}{\lambda_{0}} \sum_{j=1}^{\infty} \lambda_{0}^{-j} j K_{j}, \\
& \mu_{0}\left(\lambda_{0}\right)=\frac{1}{\lambda_{0}} \sum_{j=1}^{\infty} \lambda_{0}^{-j} j\left|K_{j}\right| .
\end{aligned}
$$

From the property (2.11) and the hypothesis that $\left(K_{n}\right)_{n \in \mathrm{N}-\{0\}}$ is not eventually identically zero it follows that

$$
0<\mu_{0}\left(\lambda_{0}\right)<1
$$

So, since $\left|\gamma_{0}\left(\lambda_{0}\right)\right| \leq \mu_{0}\left(\lambda_{0}\right)$, we have $-1<\gamma_{0}\left(\lambda_{0}\right)<1$, namely

$$
0<1+\gamma_{0}\left(\lambda_{0}\right)<2
$$

If $\left(K_{n}\right)_{n \in \mathbf{N}-\{0\}}$ is assumed to be nonpositive, then, by the fact that $\left(K_{n}\right)_{n \in \mathbf{N}-\{0\}}$ is not eventually identically zero, the property $(2.11)$ is equivalent to $-1<\gamma_{0}\left(\lambda_{0}\right)<0$, that is,

$$
0<1+\gamma_{0}\left(\lambda_{0}\right)<1
$$

Furthermore, we put

$$
\Theta_{0}\left(\lambda_{0}\right)=\frac{\left[1+\mu_{0}\left(\lambda_{0}\right)\right]^{2}}{1+\gamma_{0}\left(\lambda_{0}\right)}+\mu_{0}\left(\lambda_{0}\right)
$$

and we see that $\Theta_{0}\left(\lambda_{0}\right)$ is a real number with

$$
\Theta_{0}\left(\lambda_{0}\right)>1
$$

We notice that, in the special case of (2.3), the constants $\gamma\left(\lambda_{0}\right), \mu\left(\lambda_{0}\right)$, and $\Theta\left(\lambda_{0}\right)$, which are defined in the general case of (2.2), are equal to $\gamma_{0}\left(\lambda_{0}\right), \mu_{0}\left(\lambda_{0}\right)$, and $\Theta_{0}\left(\lambda_{0}\right)$, respectively. 
Next, consider again a positive root $\lambda_{0}$ of the characteristic equation (2.8) with the property (2.10), and let $\phi=\left(\phi_{n}\right)_{n \in \mathbf{Z}^{-}}$be an initial sequence in $S\left(\lambda_{0}\right)$. We define

$$
\begin{aligned}
L\left(\lambda_{0} ; \phi\right)= & \phi_{0}+\sum_{j=1}^{\infty} G_{j}\left[\phi_{-j}-\left(1-\frac{1}{\lambda_{0}}\right) \lambda_{0}^{-j}\left(\sum_{r=-j}^{-1} \lambda_{0}^{-r} \phi_{r}\right)\right] \\
& +\frac{1}{\lambda_{0}} \sum_{j=1}^{\infty} \lambda_{0}^{-j} K_{j}\left(\sum_{r=-j}^{-1} \lambda_{0}^{-r} \phi_{r}\right), \\
M\left(\lambda_{0} ; \phi\right)= & \sup _{n \in \mathbf{Z}^{-}}\left|\lambda_{0}^{-n} \phi_{n}-\frac{L\left(\lambda_{0} ; \phi\right)}{1+\gamma\left(\lambda_{0}\right)}\right| .
\end{aligned}
$$

From the property (2.10) and the definition of $S\left(\lambda_{0}\right)$ it follows that $L\left(\lambda_{0} ; \phi\right)$ is a real number. Moreover, by the definition of $S\left(\lambda_{0}\right), M\left(\lambda_{0} ; \phi\right)$ is a nonnegative constant.

Let us concentrate on the special case of (2.3) and consider a positive root $\lambda_{0}$ of the characteristic equation (2.9) with the property (2.11) and an initial sequence $\phi=$ $\left(\phi_{n}\right)_{n \in \mathbf{Z}^{-}}$in $S\left(\lambda_{0}\right)$. In this special case, we have the constants

$$
\begin{gathered}
L_{0}\left(\lambda_{0} ; \phi\right)=\phi_{0}+\frac{1}{\lambda_{0}} \sum_{j=1}^{\infty} \lambda_{0}^{-j} K_{j}\left(\sum_{r=-j}^{-1} \lambda_{0}^{-r} \phi_{r}\right), \\
M_{0}\left(\lambda_{0} ; \phi\right)=\sup _{n \in \mathbf{Z}^{-}}\left|\lambda_{0}^{-n} \phi_{n}-\frac{L_{0}\left(\lambda_{0} ; \phi\right)}{1+\gamma_{0}\left(\lambda_{0}\right)}\right|
\end{gathered}
$$

instead of the constants $L\left(\lambda_{0} ; \phi\right)$ and $M\left(\lambda_{0} ; \phi\right)$ considered in the general case of $(2.2)$. Property (2.11) and the definition of $S\left(\lambda_{0}\right)$ guarantee that $L_{0}\left(\lambda_{0} ; \phi\right)$ is a real number, and the definition of $S\left(\lambda_{0}\right)$ ensures that $M_{0}\left(\lambda_{0} ; \phi\right)$ is a nonnegative constant.

Another notation used in the paper is the following one

$$
N\left(\lambda_{0} ; \phi\right)=\sup _{n \in \mathbf{Z}^{-}}\left(\lambda_{0}^{-n}\left|\phi_{n}\right|\right)
$$

for each positive root $\lambda_{0}$ of the characteristic equation (2.8) (resp., (2.9)) with the property (2.10) (resp., (2.11)) and for any initial sequence $\phi=\left(\phi_{n}\right)_{n \in \mathbf{Z}^{-}}$in $S\left(\lambda_{0}\right)$. Clearly, $N\left(\lambda_{0} ; \phi\right)$ is a nonnegative constant.

Furthermore, let $\lambda_{0}$ be a positive root of the characteristic equation (2.8) with the property (2.10) and $\lambda_{1}$ be a positive root of (2.8) with $\lambda_{1}<\lambda_{0}$. Let also $\phi=\left(\phi_{n}\right)_{n \in \mathbf{Z}^{-}}$be an initial sequence in $S\left(\lambda_{0}\right)$. We set

$$
\begin{aligned}
& U\left(\lambda_{0}, \lambda_{1} ; \phi\right)=\inf _{n \in \mathbf{Z}^{-}}\left\{\lambda_{1}^{-n}\left[\phi_{n}-\frac{L\left(\lambda_{0} ; \phi\right)}{1+\gamma\left(\lambda_{0}\right)} \lambda_{0}^{n}\right]\right\}, \\
& V\left(\lambda_{0}, \lambda_{1} ; \phi\right)=\sup _{n \in \mathbf{Z}^{-}}\left\{\lambda_{1}^{-n}\left[\phi_{n}-\frac{L\left(\lambda_{0} ; \phi\right)}{1+\gamma\left(\lambda_{0}\right)} \lambda_{0}^{n}\right]\right\} .
\end{aligned}
$$

From the definition of $S\left(\lambda_{0}\right)$ and the hypothesis that $\lambda_{1}<\lambda_{0}$ it follows that $U\left(\lambda_{0}, \lambda_{1} ; \phi\right)$ and $V\left(\lambda_{0}, \lambda_{1} ; \phi\right)$ are real constants. 
In particular, consider the special case of (2.3). Let $\lambda_{0}$ be a positive root of the characteristic equation (2.9) with the property (2.11) and $\lambda_{1}$ be a positive root of (2.9) with $\lambda_{1}<\lambda_{0}$ as well as let $\phi=\left(\phi_{n}\right)_{n \in \mathbf{Z}^{-}}$be an initial sequence in $S\left(\lambda_{0}\right)$. In this special case, we consider the real constants

$$
\begin{aligned}
& U_{0}\left(\lambda_{0}, \lambda_{1} ; \phi\right)=\inf _{n \in \mathbf{Z}^{-}}\left\{\lambda_{1}^{-n}\left[\phi_{n}-\frac{L_{0}\left(\lambda_{0} ; \phi\right)}{1+\gamma_{0}\left(\lambda_{0}\right)} \lambda_{0}^{n}\right]\right\}, \\
& V_{0}\left(\lambda_{0}, \lambda_{1} ; \phi\right)=\sup _{n \in \mathbf{Z}^{-}}\left\{\lambda_{1}^{-n}\left[\phi_{n}-\frac{L_{0}\left(\lambda_{0} ; \phi\right)}{1+\gamma_{0}\left(\lambda_{0}\right)} \lambda_{0}^{n}\right]\right\}
\end{aligned}
$$

in place of $U\left(\lambda_{0}, \lambda_{1} ; \phi\right)$ and $V\left(\lambda_{0}, \lambda_{1} ; \phi\right)$ considered in the general case of (2.2).

Before closing this section, we will give three well-known definitions. The trivial solution of (2.2) (resp., of (2.3)) is said to be stable (at 0 ) if, for each $\epsilon>0$, there exists $\delta \equiv \delta(\epsilon)>0$ such that, for any $\phi=\left(\phi_{n}\right)_{n \in \mathbf{Z}^{-}}$in $S$ with $\|\phi\| \equiv \sup _{n \in \mathbf{Z}^{-}}\left|\phi_{n}\right|<\delta$, the solution $\left(x_{n}\right)_{n \in \mathbf{Z}}$ of (2.2) and (2.7) (resp., of (2.3) and (2.7)) satisfies $\left|x_{n}\right|<\epsilon$ for all $n \in \mathbf{Z}$. Also, the trivial solution of (2.2) (resp., of (2.3)) is called asymptotically stable (at 0 ) if it is stable (at 0 ) in the above sense and, in addition, there exists $\delta_{0}>0$ such that, for any $\phi=\left(\phi_{n}\right)_{n \in \mathbf{Z}^{-}}$in $S$ with $\|\phi\|<\delta_{0}$, the solution $\left(x_{n}\right)_{n \in \mathbf{Z}}$ of (2.2) and (2.7) (resp., of (2.3) and (2.7)) satisfies $\lim _{n \rightarrow \infty} x_{n}=0$. Moreover, the trivial solution of (2.2) (resp., of (2.3)) is called exponentially stable (at 0 ) if there exist positive constants $\Lambda$ and $\eta<1$ such that, for any $\phi=\left(\phi_{n}\right)_{n \in \mathbf{Z}^{-}}$in $S$ with $\|\phi\|<\infty$, the solution $\left(x_{n}\right)_{n \in \mathbf{Z}}$ of (2.2) and (2.7) (resp., of (2.3) and (2.7)) satisfies $\left|x_{n}\right| \leq \Lambda \eta^{n}\|\phi\|$ for all $n \in \mathbf{N}$ (see Elaydi and Murakami [9]).

\section{Statement of the main results}

Our first main result is Theorem 3.1 below, which establishes a useful inequality for solutions of the neutral Volterra difference equation (2.2). The application of Theorem 3.1 to the special case of the (non-neutral) Volterra difference equation (2.3) leads to Theorem 3.2 below.

THeOREM 3.1. Let $\lambda_{0}$ be a positive root of the characteristic equation (2.8) with the property (2.10). Then, for any $\phi=\left(\phi_{n}\right)_{n \in \mathbf{Z}^{-}}$in $S\left(\lambda_{0}\right)$, the solution $\left(x_{n}\right)_{n \in \mathbf{Z}}$ of (2.2) and (2.7) satisfies

$$
\left|\lambda_{0}^{-n} x_{n}-\frac{L\left(\lambda_{0} ; \phi\right)}{1+\gamma\left(\lambda_{0}\right)}\right| \leq \mu\left(\lambda_{0}\right) M\left(\lambda_{0} ; \phi\right) \quad \forall n \in \mathbf{N} .
$$

THeORem 3.2. Let $\lambda_{0}$ be a positive root of the characteristic equation (2.9) with the property (2.11). Then, for any $\phi=\left(\phi_{n}\right)_{n \in \mathbf{Z}^{-}}$in $S\left(\lambda_{0}\right)$, the solution $\left(x_{n}\right)_{n \in \mathrm{Z}}$ of (2.3) and (2.7) satisfies

$$
\left|\lambda_{0}^{-n} x_{n}-\frac{L_{0}\left(\lambda_{0} ; \phi\right)}{1+\gamma_{0}\left(\lambda_{0}\right)}\right| \leq \mu_{0}\left(\lambda_{0}\right) M_{0}\left(\lambda_{0} ; \phi\right) \quad \forall n \in \mathbf{N} .
$$

Theorem 3.3 below provides an estimate of solutions of the neutral Volterra difference equation (2.2) that leads to a stability criterion for the trivial solution of (2.2). By applying Theorem 3.3 to the special case of the (non-neutral) Volterra difference equation (2.3), one can be led to the subsequent theorem, that is, Theorem 3.4. 
THeOREM 3.3. Let $\lambda_{0}$ be a positive root of the characteristic equation (2.8) with the property (2.10). Then, for any $\phi=\left(\phi_{n}\right)_{n \in \mathbf{Z}^{-}}$in $S\left(\lambda_{0}\right)$, the solution $\left(x_{n}\right)_{n \in \mathbf{Z}}$ of (2.2) and (2.7) satisfies

$$
\left|x_{n}\right| \leq \Theta\left(\lambda_{0}\right) N\left(\lambda_{0} ; \phi\right) \lambda_{0}^{n} \quad \forall n \in \mathbf{N} .
$$

Moreover, the trivial solution of (2.2) is stable (at 0 ) if $\lambda_{0}=1$ and it is asymptotically stable (at 0 ) if $\lambda_{0}<1$. In addition, the trivial solution of (2.2) is exponentially stable (at 0 ) if $\lambda_{0}<1$.

THeOREM 3.4. Let $\lambda_{0}$ be a positive root of the characteristic equation (2.9) with the property (2.11). Then, for any $\phi=\left(\phi_{n}\right)_{n \in \mathbf{Z}^{-}}$in $S\left(\lambda_{0}\right)$, the solution $\left(x_{n}\right)_{n \in \mathbf{Z}}$ of (2.3) and (2.7) satisfies

$$
\left|x_{n}\right| \leq \Theta_{0}\left(\lambda_{0}\right) N\left(\lambda_{0} ; \phi\right) \lambda_{0}^{n} \quad \forall n \in \mathbf{N} .
$$

Moreover, the trivial solution of (2.3) is stable (at 0 ) if $\lambda_{0}=1$ and it is asymptotically stable (at 0 ) if $\lambda_{0}<1$. In addition, the trivial solution of (2.3) is exponentially stable (at 0 ) if $\lambda_{0}<1$.

It must be noted that Theorems 3.2 and 3.4 for the (non-neutral) Volterra difference equation (2.3) can be considered as substiantally improved versions of the main results of the previous authors' paper [32]. One can easily see the connection between Theorems 3.2 and 3.4 , and the main results in [32].

The following lemma, that is, Lemma 3.5, gives sufficient conditions for the characteristic equation (2.8) to have a (unique) root $\lambda_{0}$ with the property $(2.10)$. The specialization of Lemma 3.5 to the special case of the characteristic equation (2.9) is formulated below as Lemma 3.6. We notice that Lemma 3.6 has been previously proved in the authors' paper [32].

Lemma 3.5. Assume that there exists a positive real number $\gamma$ such that

$$
\begin{gathered}
\sum_{j=1}^{\infty} \gamma^{-j}\left|G_{j}\right|<\infty, \quad \sum_{j=1}^{\infty} \gamma^{-j}\left|K_{j}\right|<\infty, \\
(1-\gamma) \sum_{j=1}^{\infty} \gamma^{-j} G_{j}+\sum_{j=1}^{\infty} \gamma^{-j} K_{j}>\gamma-1-a, \\
\sum_{j=1}^{\infty} \gamma^{-j}\left[1+\left(1+\frac{1}{\gamma}\right) j\right]\left|G_{j}\right|+\frac{1}{\gamma} \sum_{j=1}^{\infty} \gamma^{-j} j\left|K_{j}\right| \leq 1 .
\end{gathered}
$$

Then, in the interval $(\gamma, \infty)$, the characteristic equation (2.8) admits a unique root $\lambda_{0}$; this root has the property (2.10).

Lemma 3.6. Assume that there exists a positive real number $\gamma$ such that

$$
\begin{gathered}
\sum_{j=1}^{\infty} \gamma^{-j}\left|K_{j}\right|<\infty, \\
\sum_{j=1}^{\infty} \gamma^{-j} K_{j}>\gamma-1-a, \\
\frac{1}{\gamma} \sum_{j=1}^{\infty} \gamma^{-j} j\left|K_{j}\right| \leq 1 .
\end{gathered}
$$


Then, in the interval $(\gamma, \infty)$, the characteristic equation (2.9) admits a unique root $\lambda_{0}$; this root has the property (2.11).

Theorem 3.7 and Corollary 3.8 below concern the behavior of solutions of the neutral Volterra difference equation (2.2), while Theorem 3.9 and Corollary 3.10 below are dealing with the behavior of solutions of the (non-neutral) Volterra difference equation (2.3).

Theorem 3.7. Suppose that $\left(G_{n}\right)_{n \in \mathbf{N}-\{0\}}$ and $\left(K_{n}\right)_{n \in \mathbf{N}-\{0\}}$ are nonpositive. Let $\lambda_{0}$ be a positive root of the characteristic equation (2.8) with $\lambda_{0} \leq 1$ and with the property (2.10). Let also $\lambda_{1}$ be a positive root of (2.8) with $\lambda_{1}<\lambda_{0}$. Then, for any $\phi=\left(\phi_{n}\right)_{n \in Z^{-}}$in $S\left(\lambda_{0}\right)$, the solution $\left(x_{n}\right)_{n \in \mathrm{Z}}$ of (2.2) and (2.7) satisfies

$$
U\left(\lambda_{0}, \lambda_{1} ; \phi\right) \leq \lambda_{1}^{-n}\left[x_{n}-\frac{L\left(\lambda_{0} ; \phi\right)}{1+\gamma\left(\lambda_{0}\right)} \lambda_{0}^{n}\right] \leq V\left(\lambda_{0}, \lambda_{1} ; \phi\right) \quad \forall n \in \mathbf{N} .
$$

We immediately observe that the double inequality in the conclusion of Theorem 3.7 can equivalently be written as follows

$$
U\left(\lambda_{0}, \lambda_{1} ; \phi\right)\left(\frac{\lambda_{1}}{\lambda_{0}}\right)^{n} \leq \lambda_{0}^{-n} x_{n}-\frac{L\left(\lambda_{0} ; \phi\right)}{1+\gamma\left(\lambda_{0}\right)} \leq V\left(\lambda_{0}, \lambda_{1} ; \phi\right)\left(\frac{\lambda_{1}}{\lambda_{0}}\right)^{n} \quad \text { for } n \in \mathbf{N} .
$$

Consequently, since $\lambda_{1}<\lambda_{0}$, we obtain

$$
\lim _{n \rightarrow \infty}\left(\lambda_{0}^{-n} x_{n}\right)=\frac{L\left(\lambda_{0} ; \phi\right)}{1+\gamma\left(\lambda_{0}\right)}
$$

which establishes the following corollary.

Corollary 3.8. Suppose that $\left(G_{n}\right)_{n \in \mathbf{N}-\{0\}}$ and $\left(K_{n}\right)_{n \in \mathbf{N}-\{0\}}$ are nonpositive. Let $\lambda_{0}$ be a positive root of the characteristic equation (2.8) with $\lambda_{0} \leq 1$ and with the property (2.10). Assume that (2.8) has another positive root less than $\lambda_{0}$. Then, for any $\phi=\left(\phi_{n}\right)_{n \in \mathbf{Z}^{-}}$in $S\left(\lambda_{0}\right)$, the solution $\left(x_{n}\right)_{n \in \mathrm{Z}}$ of (2.2) and (2.7) satisfies

$$
\lim _{n \rightarrow \infty}\left(\lambda_{0}^{-n} x_{n}\right)=\frac{L\left(\lambda_{0} ; \phi\right)}{1+\gamma\left(\lambda_{0}\right)}
$$

Theorem 3.9. Suppose that $\left(K_{n}\right)_{n \in \mathbf{N}-\{0\}}$ is nompositive. Let $\lambda_{0}$ be a positive root of the characteristic equation (2.9) with the property (2.11). Let also $\lambda_{1}$ be a positive root of (2.9) with $\lambda_{1}<\lambda_{0}$. Then, for any $\phi=\left(\phi_{n}\right)_{n \in Z^{-}}$in $S\left(\lambda_{0}\right)$, the solution $\left(x_{n}\right)_{n \in Z}$ of (2.3) and (2.7) satisfies

$$
U_{0}\left(\lambda_{0}, \lambda_{1} ; \phi\right) \leq \lambda_{1}^{-n}\left[x_{n}-\frac{L_{0}\left(\lambda_{0} ; \phi\right)}{1+\gamma_{0}\left(\lambda_{0}\right)} \lambda_{0}^{n}\right] \leq V_{0}\left(\lambda_{0}, \lambda_{1} ; \phi\right) \quad \forall n \in \mathbf{N}
$$

We see that the double inequality in the conclusion of Theorem 3.9 is equivalently written as

$$
U_{0}\left(\lambda_{0}, \lambda_{1} ; \phi\right)\left(\frac{\lambda_{1}}{\lambda_{0}}\right)^{n} \leq \lambda_{0}^{-n} x_{n}-\frac{L_{0}\left(\lambda_{0} ; \phi\right)}{1+\gamma_{0}\left(\lambda_{0}\right)} \leq V_{0}\left(\lambda_{0}, \lambda_{1} ; \phi\right)\left(\frac{\lambda_{1}}{\lambda_{0}}\right)^{n} \quad \text { for } n \in \mathbf{N}
$$


10 Volterra difference equations with infinite delay

So, as $\lambda_{1}<\lambda_{0}$, we have

$$
\lim _{n \rightarrow \infty}\left(\lambda_{0}^{-n} x_{n}\right)=\frac{L_{0}\left(\lambda_{0} ; \phi\right)}{1+\gamma_{0}\left(\lambda_{0}\right)}
$$

This proves the following corollary.

Corollary 3.10. Suppose that $\left(K_{n}\right)_{n \in \mathrm{N}-\{0\}}$ is nompositive. Let $\lambda_{0}$ be a positive root of the characteristic equation (2.9) with the property (2.11). Assume that (2.9) has another positive root less than $\lambda_{0}$. Then, for any $\phi=\left(\phi_{n}\right)_{n \in \mathrm{Z}^{-}}$in $S\left(\lambda_{0}\right)$, the solution $\left(x_{n}\right)_{n \in \mathrm{Z}}$ of (2.3) and (2.7) satisfies

$$
\lim _{n \rightarrow \infty}\left(\lambda_{0}^{-n} x_{n}\right)=\frac{L_{0}\left(\lambda_{0} ; \phi\right)}{1+\gamma_{0}\left(\lambda_{0}\right)}
$$

Now, we state two propositions (Propositions 3.11 and 3.12) as well as two lemmas (Lemmas 3.13 and 3.14). Proposition 3.11 and Lemma 3.13 give some useful information about the positive roots of the characteristic equation (2.8), while Proposition 3.12 and Lemma 3.14 are concerned with the special case of the positive roots of the characteristic equation (2.9).

Proposition 3.11. Suppose that $\left(G_{n}\right)_{n \in \mathbf{N}-\{0\}}$ and $\left(K_{n}\right)_{n \in \mathbf{N}-\{0\}}$ are nonpositive. Let $\lambda_{0}$ be a positive root of the characteristic equation (2.8) with $\lambda_{0} \leq 1$. If there exists another positive root $\lambda_{1}$ of (2.8) with $\lambda_{1}<\lambda_{0}$ such that

$$
\sum_{j=1}^{\infty} \lambda_{1}^{-j} j\left|G_{j}\right|<\infty, \quad \sum_{j=1}^{\infty} \lambda_{1}^{-j} j\left|K_{j}\right|<\infty,
$$

then $\lambda_{0}$ has the property (2.10).

Proposition 3.12. Suppose that $\left(K_{n}\right)_{n \in \mathrm{N}-\{0\}}$ is nonpositive. Let $\lambda_{0}$ be a positive root of the characteristic equation (2.9). If there exists another positive root $\lambda_{1}$ of (2.9) with $\lambda_{1}<\lambda_{0}$ such that

$$
\sum_{j=1}^{\infty} \lambda_{1}^{-j} j\left|K_{j}\right|<\infty
$$

then $\lambda_{0}$ has the property (2.11).

Lemma 3.13. Suppose that $\left(G_{n}\right)_{n \in \mathbf{N}-\{0\}}$ and $\left(K_{n}\right)_{n \in \mathbf{N}-\{0\}}$ are nonpositive.

(I) If $a=0$, then $\lambda=1$ is not a root of the characteristic equation (2.8).

(II) Assume that $a=0$ and that

$$
\sum_{j=1}^{\infty}\left|G_{j}\right| \leq 1
$$

Then, in the interval $(1, \infty)$, the characteristic equation (2.8) has no roots. 
(III) Assume that

$$
\begin{gathered}
\sum_{j=1}^{\infty} j\left|G_{j}\right|<\infty, \\
\sum_{j=1}^{\infty}\left|G_{j}\right|+\sum_{j=1}^{\infty} j\left|K_{j}\right| \leq 1, \\
\sum_{j=1}^{\infty}\left|K_{j}\right| \geq a .
\end{gathered}
$$

Then, in the interval $(1, \infty)$, the characteristic equation (2.8) has no roots.

(IV) Assume that (3.22) holds, and let there exist a positive real number $\gamma$ with $\gamma<1$ and $\gamma<a+1$ so that

$$
\begin{gathered}
\sum_{j=1}^{\infty} \gamma^{-j} j\left|G_{j}\right|<\infty, \quad \sum_{j=1}^{\infty} \gamma^{-j} j\left|K_{j}\right|<\infty, \\
(1-\gamma) \sum_{j=1}^{\infty} \gamma^{-j}\left|G_{j}\right|+\sum_{j=1}^{\infty} \gamma^{-j}\left|K_{j}\right|>a+1-\gamma .
\end{gathered}
$$

Moreover, assume that there exists a real number $\delta$ with $\delta>0$ and $a<\delta<a+1-\gamma$ such that

$$
(\delta-a) \sum_{j=1}^{\infty}(a+1-\delta)^{-j}\left|G_{j}\right|+\sum_{j=1}^{\infty}(a+1-\delta)^{-j}\left|K_{j}\right|<\delta .
$$

Then: (i) $\lambda=a+1-\delta$ is not a root of the characteristic equation (2.8). (ii) $\lambda=\gamma$ is not a root of (2.8). (iii) In the interval $(a+1-\delta, 1],(2.8)$ has a unique root. (iv) In the interval $(\gamma, a+1-\delta),(2.8)$ has a unique root. (Note: We have $\delta>0$ and $\gamma<a+1-\delta<1$.)

Lemma 3.14. Suppose that $\left(K_{n}\right)_{n \in \mathbf{N}-\{0\}}$ is nonpositive.

(I) $a>-1$ is a necessary condition for the characteristic equation (2.9) to have at least one positive root.

(II) The characteristic equation (2.9) has no positive roots greater than or equal to $a+1$.

(III) Let $a>-1$ and let there exist a positive real number $\gamma$ with $\gamma<a+1$ so that

$$
\begin{gathered}
\sum_{j=1}^{\infty} \gamma^{-j} j\left|K_{j}\right|<\infty, \\
\sum_{j=1}^{\infty} \gamma^{-j}\left|K_{j}\right|>a+1-\gamma .
\end{gathered}
$$


Moreover, assume that there exists a real number $\delta$ with $0<\delta<a+1-\gamma$ such that

$$
\sum_{j=1}^{\infty}(a+1-\delta)^{-j}\left|K_{j}\right|<\delta
$$

Then: (i) $\lambda=a+1-\delta$ is not a root of the characteristic equation (2.9). (ii) $\lambda=\gamma$ is not a root of (2.9). (iii) In the interval $(a+1-\delta, a+1)$, (2.9) has a unique root. (iv) In the interval $(\gamma, a+1-\delta)$, (2.9) has a unique root. (Note: We have $\gamma<a+1-\delta<a+1)$.

It is an open problem to examine if Theorem 3.7, Corollary 3.8, and Proposition 3.11 remain valid without the restriction that the root $\lambda_{0}$ of the characteristic equation (2.8) satisfies $\lambda_{0} \leq 1$. Such a restriction is not a necessity in the non-neutral case (i.e., in Theorem 3.9, Corollary 3.10, and Proposition 3.12).

The neutral Volterra difference equation with infinite delay (2.2) can be considered as the discrete version of the neutral Volterra integrodifferential equation with unbounded delay

$$
\left[x(t)+\int_{-\infty}^{t} G(t-s) x(s) d s\right]^{\prime}=a x(t)+\int_{-\infty}^{t} K(t-s) x(s) d s
$$

where $a$ is a real number, $G$ and $K$ are continuous real-valued functions on the interval $[0, \infty)$, and $K$ is assumed to be not eventually identically zero. In particular, the (nonneutral) Volterra difference equation with infinite delay (2.3) can be viewed as the discrete version of the (non-neutral) Volterra integrodifferential equation with unbounded delay

$$
x^{\prime}(t)=a x(t)+\int_{-\infty}^{t} K(t-s) x(s) d s
$$

The results obtained in this paper should be looked upon as the discrete analogues of the ones given by Kordonis and Philos [19], Kordonis et al. [21], and Philos and Purnaras [33], for the neutral Volterra integrodifferential equation with unbounded delay (3.29) and, especially, for the (non-neutral) Volterra integrodifferential equation with unbounded delay (3.30).

\section{Proofs of the main results}

Proof of Theorem 3.1. Let $\phi=\left(\phi_{n}\right)_{n \in \mathbf{Z}^{-}}$be an initial sequence in $S\left(\lambda_{0}\right)$, and $\left(x_{n}\right)_{n \in \mathbf{Z}}$ be the solution of (2.2) and (2.7).

Define

$$
y_{n}=\lambda_{0}^{-n} x_{n} \quad \text { for } n \in \mathbf{Z} \text {. }
$$


Then, for each $n \in \mathbf{N}$, we obtain

$$
\begin{aligned}
& \Delta\left(x_{n}+\sum_{j=1}^{\infty} G_{j} x_{n-j}\right)-a x_{n}-\sum_{j=1}^{\infty} K_{j} x_{n-j} \\
& =\Delta\left[\lambda_{0}^{n}\left(y_{n}+\sum_{j=1}^{\infty} \lambda_{0}^{-j} G_{j} y_{n-j}\right)\right]-a \lambda_{0}^{n} y_{n}-\lambda_{0}^{n} \sum_{j=1}^{\infty} \lambda_{0}^{-j} K_{j} y_{n-j} \\
& =\lambda_{0}^{n}\left[\lambda_{0} \Delta\left(y_{n}+\sum_{j=1}^{\infty} \lambda_{0}^{-j} G_{j} y_{n-j}\right)+\left(\lambda_{0}-1-a\right) y_{n}\right. \\
& \left.+\left(\lambda_{0}-1\right) \sum_{j=1}^{\infty} \lambda_{0}^{-j} G_{j} y_{n-j}-\sum_{j=1}^{\infty} \lambda_{0}^{-j} K_{j} y_{n-j}\right] \\
& =\lambda_{0}^{n}\left[\lambda_{0} \Delta\left(y_{n}+\sum_{j=1}^{\infty} \lambda_{0}^{-j} G_{j} y_{n-j}\right)-\left(\lambda_{0}-1\right)\left(\sum_{j=1}^{\infty} \lambda_{0}^{-j} G_{j}\right) y_{n}\right. \\
& \left.+\left(\sum_{j=1}^{\infty} \lambda_{0}^{-j} K_{j}\right) y_{n}+\left(\lambda_{0}-1\right) \sum_{j=1}^{\infty} \lambda_{0}^{-j} G_{j} y_{n-j}-\sum_{j=1}^{\infty} \lambda_{0}^{-j} K_{j} y_{n-j}\right] \\
& =\lambda_{0}^{n}\left[\lambda_{0} \Delta\left(y_{n}+\sum_{j=1}^{\infty} \lambda_{0}^{-j} G_{j} y_{n-j}\right)-\left(\lambda_{0}-1\right) \sum_{j=1}^{\infty} \lambda_{0}^{-j} G_{j}\left(y_{n}-y_{n-j}\right)\right. \\
& \left.+\sum_{j=1}^{\infty} \lambda_{0}^{-j} K_{j}\left(y_{n}-y_{n-j}\right)\right] \text {. }
\end{aligned}
$$

So, $\left(x_{n}\right)_{n \in \mathbf{Z}}$ satisfies (2.2) for $n \in \mathbf{N}$ if and only if $\left(y_{n}\right)_{n \in \mathbf{Z}}$ satisfies

$$
\begin{aligned}
& \Delta\left(y_{n}+\sum_{j=1}^{\infty} \lambda_{0}^{-j} G_{j} y_{n-j}\right) \\
& \quad=\left(1-\frac{1}{\lambda_{0}}\right) \sum_{j=1}^{\infty} \lambda_{0}^{-j} G_{j}\left(y_{n}-y_{n-j}\right)-\frac{1}{\lambda_{0}} \sum_{j=1}^{\infty} \lambda_{0}^{-j} K_{j}\left(y_{n}-y_{n-j}\right) \quad \text { for } n \in \mathbf{N} .
\end{aligned}
$$

Moreover, the initial condition (2.7) can equivalently be written as

$$
y_{n}=\lambda_{0}^{-n} \phi_{n} \quad \text { for } n \in \mathbf{Z}^{-} .
$$

Furthermore, we see that (4.3) becomes

$$
\begin{aligned}
& \Delta\left(y_{n}+\sum_{j=1}^{\infty} \lambda_{0}^{-j} G_{j} y_{n-j}\right) \\
& \quad=\Delta\left[\left(1-\frac{1}{\lambda_{0}}\right) \sum_{j=1}^{\infty} \lambda_{0}^{-j} G_{j}\left(\sum_{r=n-j}^{n-1} y_{r}\right)-\frac{1}{\lambda_{0}} \sum_{j=1}^{\infty} \lambda_{0}^{-j} K_{j}\left(\sum_{r=n-j}^{n-1} y_{r}\right)\right]
\end{aligned}
$$


14 Volterra difference equations with infinite delay

for $n \in \mathbf{N}$. Thus, we have

$$
\begin{aligned}
y_{n} & +\sum_{j=1}^{\infty} \lambda_{0}^{-j} G_{j} y_{n-j} \\
& =\left(1-\frac{1}{\lambda_{0}}\right) \sum_{j=1}^{\infty} \lambda_{0}^{-j} G_{j}\left(\sum_{r=n-j}^{n-1} y_{r}\right)-\frac{1}{\lambda_{0}} \sum_{j=1}^{\infty} \lambda_{0}^{-j} K_{j}\left(\sum_{r=n-j}^{n-1} y_{r}\right)+\Lambda
\end{aligned}
$$

for every $n \in \mathbf{N}$, where $\Lambda$ is a real constant. But, by using (4.4) and taking into account the definition of $L\left(\lambda_{0} ; \phi\right)$, we can immediately verify that $\Lambda=L\left(\lambda_{0} ; \phi\right)$. Hence, (4.3) takes the following equivalent form

$$
\begin{aligned}
y_{n} & +\sum_{j=1}^{\infty} \lambda_{0}^{-j} G_{j} y_{n-j} \\
& =\left(1-\frac{1}{\lambda_{0}}\right) \sum_{j=1}^{\infty} \lambda_{0}^{-j} G_{j}\left(\sum_{r=n-j}^{n-1} y_{r}\right)-\frac{1}{\lambda_{0}} \sum_{j=1}^{\infty} \lambda_{0}^{-j} K_{j}\left(\sum_{r=n-j}^{n-1} y_{r}\right)+L\left(\lambda_{0} ; \phi\right) \quad \text { for } n \in \mathbf{N} .
\end{aligned}
$$

Next, we set

$$
z_{n}=y_{n}-\frac{L\left(\lambda_{0} ; \phi\right)}{1+\gamma\left(\lambda_{0}\right)} \quad \text { for } n \in \mathbf{Z} .
$$

Then, we take into account the definition of $\gamma\left(\lambda_{0}\right)$ to show that (4.7) may equivalently be written as follows

$$
\begin{aligned}
z_{n} & +\sum_{j=1}^{\infty} \lambda_{0}^{-j} G_{j} z_{n-j} \\
& =\left(1-\frac{1}{\lambda_{0}}\right) \sum_{j=1}^{\infty} \lambda_{0}^{-j} G_{j}\left(\sum_{r=n-j}^{n-1} z_{r}\right)-\frac{1}{\lambda_{0}} \sum_{j=1}^{\infty} \lambda_{0}^{-j} K_{j}\left(\sum_{r=n-j}^{n-1} z_{r}\right) \quad \text { for } n \in \mathbf{N} .
\end{aligned}
$$

On the other hand, the initial condition (4.4) becomes

$$
z_{n}=\lambda_{0}^{-n} \phi_{n}-\frac{L\left(\lambda_{0} ; \phi\right)}{1+\gamma\left(\lambda_{0}\right)} \quad \text { for } n \in \mathbf{Z}^{-}
$$

Now, by taking into account the definitions of $\left(y_{n}\right)_{n \in Z}$ and $\left(z_{n}\right)_{n \in Z}$, we conclude that what we have to prove is that $\left(z_{n}\right)_{n \in \mathrm{Z}}$ satisfies

$$
\left|z_{n}\right| \leq \mu\left(\lambda_{0}\right) M\left(\lambda_{0} ; \phi\right) \quad \forall n \in \mathbf{N}
$$

In the rest of the proof we will establish (4.11). From (4.10) and the definition of $M\left(\lambda_{0} ; \phi\right)$ it follows that

$$
\left|z_{n}\right| \leq M\left(\lambda_{0} ; \phi\right) \quad \text { for } n \in \mathbf{Z}^{-}
$$


We will show that

$$
\left|z_{n}\right| \leq M\left(\lambda_{0} ; \phi\right) \quad \forall n \in \mathbf{Z}
$$

For this purpose, let us consider an arbitrary real number $\epsilon>0$. Then (4.12) guarantees that

$$
\left|z_{n}\right|<M\left(\lambda_{0} ; \phi\right)+\epsilon \quad \text { for } n \in \mathbf{Z}^{-} \text {. }
$$

We claim that

$$
\left|z_{n}\right|<M\left(\lambda_{0} ; \phi\right)+\epsilon \quad \text { for every } n \in \mathbf{Z} .
$$

Otherwise, because of (4.14), there exists an integer $n_{0}>0$ so that

$$
\left|z_{n}\right|<M\left(\lambda_{0} ; \phi\right)+\epsilon \quad \text { for } n \in \mathbf{Z} \text { with } n \leq n_{0}-1,\left|z_{n_{0}}\right| \geq M\left(\lambda_{0} ; \phi\right)+\epsilon \text {. }
$$

Then, by taking into account the definition of $\mu\left(\lambda_{0}\right)$ and the fact that $0<\mu\left(\lambda_{0}\right)<1$, from (4.9) we obtain

$$
\begin{aligned}
M\left(\lambda_{0} ; \phi\right) & +\epsilon \\
\leq & \left|z_{n_{0}}\right| \\
\leq & \sum_{j=1}^{\infty} \lambda_{0}^{-j}\left|G_{j}\right|\left|z_{n_{0}-j}\right|+\left|1-\frac{1}{\lambda_{0}}\right| \sum_{j=1}^{\infty} \lambda_{0}^{-j}\left|G_{j}\right|\left(\sum_{r=n_{0}-j}^{n_{0}-1}\left|z_{r}\right|\right) \\
& +\frac{1}{\lambda_{0}} \sum_{j=1}^{\infty} \lambda_{0}^{-j}\left|K_{j}\right|\left(\sum_{r=n_{0}-j}^{n_{0}-1}\left|z_{r}\right|\right) \\
\leq & {\left[\sum_{j=1}^{\infty} \lambda_{0}^{-j}\left(1+\left|1-\frac{1}{\lambda_{0}}\right| j\right)\left|G_{j}\right|+\frac{1}{\lambda_{0}} \sum_{j=1}^{\infty} \lambda_{0}^{-j} j\left|K_{j}\right|\right]\left[M\left(\lambda_{0} ; \phi\right)+\epsilon\right] } \\
\equiv & \mu\left(\lambda_{0}\right)\left[M\left(\lambda_{0} ; \phi\right)+\epsilon\right]<M\left(\lambda_{0} ; \phi\right)+\epsilon .
\end{aligned}
$$

This is a contradiction and consequently our claim is true, that is, (4.15) holds true. Since (4.15) is fulfilled for all numbers $\epsilon>0$, we conclude that (4.13) is always satisfied. Finally, using (4.13) and taking again into account the definition of $\mu\left(\lambda_{0}\right)$, from (4.9) we derive, for every $n \in \mathbf{N}$,

$$
\begin{aligned}
\left|z_{n}\right| \leq & \sum_{j=1}^{\infty} \lambda_{0}^{-j}\left|G_{j}\right|\left|z_{n-j}\right|+\left|1-\frac{1}{\lambda_{0}}\right| \sum_{j=1}^{\infty} \lambda_{0}^{-j}\left|G_{j}\right|\left(\sum_{r=n-j}^{n-1}\left|z_{r}\right|\right) \\
& +\frac{1}{\lambda_{0}} \sum_{j=1}^{\infty} \lambda_{0}^{-j}\left|K_{j}\right|\left(\sum_{r=n-j}^{n-1}\left|z_{r}\right|\right) \\
\leq & {\left[\sum_{j=1}^{\infty} \lambda_{0}^{-j}\left(1+\left|1-\frac{1}{\lambda_{0}}\right| j\right)\left|G_{j}\right|+\frac{1}{\lambda_{0}} \sum_{j=1}^{\infty} \lambda_{0}^{-j} j\left|K_{j}\right|\right] M\left(\lambda_{0} ; \phi\right) } \\
\equiv & \mu\left(\lambda_{0}\right) M\left(\lambda_{0} ; \phi\right) .
\end{aligned}
$$

Consequently, (4.11) has been proved.

The proof of our theorem is complete. 
Proof of Theorem 3.3. Consider an arbitrary initial sequence $\phi=\left(\phi_{n}\right)_{n \in Z^{-}}$in $S\left(\lambda_{0}\right)$ and let $\left(x_{n}\right)_{n \in \mathbf{Z}}$ be the solution of (2.2) and (2.7). Then, by Theorem 3.1, it holds

$$
\left|\lambda_{0}^{-n} x_{n}-\frac{L\left(\lambda_{0} ; \phi\right)}{1+\gamma\left(\lambda_{0}\right)}\right| \leq \mu\left(\lambda_{0}\right) M\left(\lambda_{0} ; \phi\right) \quad \forall n \in \mathbf{N},
$$

which leads to

$$
\lambda_{0}^{-n}\left|x_{n}\right| \leq \frac{\left|L\left(\lambda_{0} ; \phi\right)\right|}{1+\gamma\left(\lambda_{0}\right)}+\mu\left(\lambda_{0}\right) M\left(\lambda_{0} ; \phi\right) \quad \text { for every } n \in \mathbf{N}
$$

On the other hand, the definitions of $M\left(\lambda_{0} ; \phi\right)$ and $N\left(\lambda_{0} ; \phi\right)$ give

$$
M\left(\lambda_{0} ; \phi\right) \leq N\left(\lambda_{0} ; \phi\right)+\frac{\left|L\left(\lambda_{0} ; \phi\right)\right|}{1+\gamma\left(\lambda_{0}\right)} .
$$

Thus, we have

$$
\lambda_{0}^{-n}\left|x_{n}\right| \leq \frac{1+\mu\left(\lambda_{0}\right)}{1+\gamma\left(\lambda_{0}\right)}\left|L\left(\lambda_{0} ; \phi\right)\right|+\mu\left(\lambda_{0}\right) N\left(\lambda_{0} ; \phi\right) \quad \text { for } n \in \mathbf{N}
$$

But, taking into account the definitions of $L\left(\lambda_{0} ; \phi\right), N\left(\lambda_{0} ; \phi\right)$, and $\mu\left(\lambda_{0}\right)$, we obtain

$$
\begin{aligned}
\left|L\left(\lambda_{0} ; \phi\right)\right| \leq & \left|\phi_{0}\right|+\sum_{j=1}^{\infty} \lambda_{0}^{-j}\left[\lambda_{0}^{-(-j)}\left|\phi_{-j}\right|+\left|1-\frac{1}{\lambda_{0}}\right|\left(\sum_{r=-j}^{-1} \lambda_{0}^{-r}\left|\phi_{r}\right|\right)\right]\left|G_{j}\right| \\
& +\frac{1}{\lambda_{0}} \sum_{j=1}^{\infty} \lambda_{0}^{-j}\left(\sum_{r=-j}^{-1} \lambda_{0}^{-r}\left|\phi_{r}\right|\right)\left|K_{j}\right| \\
\leq & {\left[1+\sum_{j=1}^{\infty} \lambda_{0}^{-j}\left(1+\left|1-\frac{1}{\lambda_{0}}\right| j\right)\left|G_{j}\right|+\frac{1}{\lambda_{0}} \sum_{j=1}^{\infty} \lambda_{0}^{-j} j\left|K_{j}\right|\right] N\left(\lambda_{0} ; \phi\right) } \\
= & {\left[1+\mu\left(\lambda_{0}\right)\right] N\left(\lambda_{0} ; \phi\right) . }
\end{aligned}
$$

This together with (4.22) give

$$
\lambda_{0}^{-n}\left|x_{n}\right| \leq\left\{\frac{\left[1+\mu\left(\lambda_{0}\right)\right]^{2}}{1+\gamma\left(\lambda_{0}\right)}+\mu\left(\lambda_{0}\right)\right\} N\left(\lambda_{0} ; \phi\right) \quad \text { for } n \in \mathbf{N}
$$

and hence, by taking into account the definition of $\Theta\left(\lambda_{0}\right)$, we have

$$
\left|x_{n}\right| \leq \Theta\left(\lambda_{0}\right) N\left(\lambda_{0} ; \phi\right) \lambda_{0}^{n} \quad \forall n \in \mathbf{N}
$$

We have thus proved the first part of the theorem.

Next, we will establish the stability criterion contained in our theorem. Assume that $\lambda_{0} \leq 1$. Consider an arbitrary bounded initial sequence $\phi=\left(\phi_{n}\right)_{n \in \mathbf{Z}^{-}}$in $S$ and define

$$
\|\phi\|=\sup _{n \in \mathbf{Z}^{-}}\left|\phi_{n}\right|
$$


As $\lambda_{0} \leq 1$, we immediately see that $\phi=\left(\phi_{n}\right)_{n \in \mathbf{Z}^{-}}$belongs to $S\left(\lambda_{0}\right)$ and, in addition, that

$$
N\left(\lambda_{0} ; \phi\right) \leq\|\phi\| \text {. }
$$

The solution $\left(x_{n}\right)_{n \in \mathrm{Z}}$ of (2.2) and (2.7) satisfies (4.25). By combining (4.25) and (4.27), we obtain

$$
\left|x_{n}\right| \leq \Theta\left(\lambda_{0}\right)\|\phi\| \lambda_{0}^{n} \quad \text { for every } n \in \mathbf{N} .
$$

Since $\lambda_{0} \leq 1$, it follows from (4.28) that

$$
\left|x_{n}\right| \leq \Theta\left(\lambda_{0}\right)\|\phi\| \quad \text { for any } n \in \mathbf{N} .
$$

Thus, as $\Theta\left(\lambda_{0}\right)>1$, we always have

$$
\left|x_{n}\right| \leq \Theta\left(\lambda_{0}\right)\|\phi\| \quad \forall n \in \mathbf{Z} .
$$

We have proved that, for any bounded initial sequence $\phi=\left(\phi_{n}\right)_{n \in Z^{-}}$in $S$, the solution $\left(x_{n}\right)_{n \in \mathbf{Z}}$ of (2.2) and (2.7) satisfies (4.28) and (4.30). From (4.30) it follows that the trivial solution of (2.2) is stable (at 0 ), provided that $\lambda_{0} \leq 1$. Furthermore, if $\lambda_{0}<1$, then (4.28) ensures that $\lim _{n \rightarrow \infty} x_{n}=0$ and hence the trivial solution of (2.2) is asymptotically stable (at 0 ). Finally, if $\lambda_{0}<1$, then it follows from (4.28) that the trivial solution of (2.2) is also exponentially stable (at 0 ).

The proof of the theorem has been finished.

Proof of Lemma 3.5. Assumption (3.5) guarantees that

$$
\sum_{j=1}^{\infty} \lambda^{-j}\left|G_{j}\right|<\infty, \quad \sum_{j=1}^{\infty} \lambda^{-j}\left|K_{j}\right|<\infty, \quad \forall \lambda \geq \gamma
$$

and hence the formula

$$
F(\lambda)=(\lambda-1)\left(1+\sum_{j=1}^{\infty} \lambda^{-j} G_{j}\right)-a-\sum_{j=1}^{\infty} \lambda^{-j} K_{j} \quad \text { for } \lambda \geq \gamma
$$

defines a continuous real-valued function on the interval $[\gamma, \infty)$. From condition (3.6) it follows that

$$
F(\gamma)<0
$$

Furthermore, for each $\lambda \geq \gamma$, we obtain

$$
\left|\sum_{j=1}^{\infty} \lambda^{-j} G_{j}\right| \leq \frac{1}{\lambda} \sum_{j=1}^{\infty} \lambda^{-j+1}\left|G_{j}\right| \leq \frac{1}{\lambda} \sum_{j=1}^{\infty} \gamma^{-j+1}\left|G_{j}\right|=\frac{\gamma}{\lambda} \sum_{j=1}^{\infty} \gamma^{-j}\left|G_{j}\right|
$$

and consequently, by the first assumption of (3.5), we have

$$
\lim _{\lambda \rightarrow \infty} \sum_{j=1}^{\infty} \lambda^{-j} G_{j}=0
$$


In a similar way, one can see that

$$
\lim _{\lambda \rightarrow \infty} \sum_{j=1}^{\infty} \lambda^{-j} K_{j}=0 .
$$

So, we immediately verify that

$$
F(\infty)=\infty
$$

Now, by using the hypothesis that $\left(K_{n}\right)_{n \in \mathrm{N}-\{0\}}$ is not eventually identically zero as well as condition (3.7), we derive for $\lambda>\gamma$

$$
\begin{aligned}
F^{\prime}(\lambda) & =1+\sum_{j=1}^{\infty} \lambda^{-j}\left[1-\left(1-\frac{1}{\lambda}\right) j\right] G_{j}+\frac{1}{\lambda} \sum_{j=1}^{\infty} \lambda^{-j} j K_{j} \\
& \geq 1-\sum_{j=1}^{\infty} \lambda^{-j}\left[1+\left(1+\frac{1}{\lambda}\right) j\right]\left|G_{j}\right|-\frac{1}{\lambda} \sum_{j=1}^{\infty} \lambda^{-j} j\left|K_{j}\right| \\
& >1-\sum_{j=1}^{\infty} \gamma^{-j}\left[1+\left(1+\frac{1}{\gamma}\right) j\right]\left|G_{j}\right|-\frac{1}{\gamma} \sum_{j=1}^{\infty} \gamma^{-j} j\left|K_{j}\right| \geq 0,
\end{aligned}
$$

which means that $F$ is strictly increasing on $(\gamma, \infty)$. This fact together with (4.33) and (4.37) guarantee that, in the interval $(\gamma, \infty)$, the equation $F(\lambda)=0$ (i.e., the characteristic equation (2.8)) has a unique root $\lambda_{0}$. Finally, by using again the hypothesis that $\left(K_{n}\right)_{n \in \mathrm{N}-\{0\}}$ is not eventually identically zero as well as condition (3.7), we get

$$
\begin{aligned}
\sum_{j=1}^{\infty} \lambda_{0}^{-j} & \left(1+\left|1-\frac{1}{\lambda_{0}}\right| j\right)\left|G_{j}\right|+\frac{1}{\lambda_{0}} \sum_{j=1}^{\infty} \lambda_{0}^{-j} j\left|K_{j}\right| \\
& \leq \sum_{j=1}^{\infty} \lambda_{0}^{-j}\left[1+\left(1+\frac{1}{\lambda_{0}}\right) j\right]\left|G_{j}\right|+\frac{1}{\lambda_{0}} \sum_{j=1}^{\infty} \lambda_{0}^{-j} j\left|K_{j}\right| \\
& <\sum_{j=1}^{\infty} \gamma^{-j}\left[1+\left(1+\frac{1}{\gamma}\right) j\right]\left|G_{j}\right|+\frac{1}{\gamma} \sum_{j=1}^{\infty} \gamma^{-j} j\left|K_{j}\right| \leq 1 .
\end{aligned}
$$

So, the root $\lambda_{0}$ of the characteristic equation (2.8) has the property (2.10). This completes the proof of the lemma.

Proof of Theorem 3.7. Let $\phi=\left(\phi_{n}\right)_{n \in \mathbf{Z}^{-}}$be an arbitrary initial sequence in $S\left(\lambda_{0}\right)$ and $\left(x_{n}\right)_{n \in \mathbf{Z}}$ be the solution of (2.2) and (2.7). Define $\left(y_{n}\right)_{n \in \mathbf{Z}}$ and $\left(z_{n}\right)_{n \in \mathbf{Z}}$ as in the proof of Theorem 3.1. As it has been shown in the proof of Theorem 3.1, the fact that $\left(x_{n}\right)_{n \in Z}$ satisfies (2.2) for $n \in \mathbf{N}$ is equivalent to the fact that $\left(z_{n}\right)_{n \in Z}$ satisfies (4.9), while the initial condition (2.7) becomes (4.10). Furthermore, set

$$
w_{n}=\left(\frac{\lambda_{0}}{\lambda_{1}}\right)^{n} z_{n} \quad \text { for } n \in \mathbf{Z} .
$$


Then it is easy to see that (4.9) can equivalently be written as follows

$$
\begin{aligned}
w_{n}+\sum_{j=1}^{\infty} \lambda_{1}^{-j} G_{j} w_{n-j}= & \left(1-\frac{1}{\lambda_{0}}\right) \sum_{j=1}^{\infty} \lambda_{0}^{-j} G_{j}\left[\sum_{r=n-j}^{n-1}\left(\frac{\lambda_{0}}{\lambda_{1}}\right)^{n-r} w_{r}\right] \\
& -\frac{1}{\lambda_{0}} \sum_{j=1}^{\infty} \lambda_{0}^{-j} K_{j}\left[\sum_{r=n-j}^{n-1}\left(\frac{\lambda_{0}}{\lambda_{1}}\right)^{n-r} w_{r}\right] \text { for } n \in \mathbf{N} .
\end{aligned}
$$

Moreover, the initial condition (4.10) is written in the following equivalent form

$$
w_{n}=\lambda_{1}^{-n}\left[\phi_{n}-\frac{L\left(\lambda_{0} ; \phi\right)}{1+\gamma\left(\lambda_{0}\right)} \lambda_{0}^{n}\right] \quad \text { for } n \in \mathbf{Z}^{-} .
$$

In view of the definitions of $\left(y_{n}\right)_{n \in \mathbf{Z}},\left(z_{n}\right)_{n \in \mathbf{Z}}$, and $\left(w_{n}\right)_{n \in \mathbf{Z}}$, we have

$$
w_{n}=\lambda_{1}^{-n}\left[x_{n}-\frac{L\left(\lambda_{0} ; \phi\right)}{1+\gamma\left(\lambda_{0}\right)} \lambda_{0}^{n}\right] \quad \text { for } n \in \mathbf{Z} \text {. }
$$

From (4.42) and the definitions of $U\left(\lambda_{0}, \lambda_{1} ; \phi\right)$ and $V\left(\lambda_{0}, \lambda_{1} ; \phi\right)$ it follows that

$$
U\left(\lambda_{0}, \lambda_{1} ; \phi\right)=\inf _{s \in \mathbf{Z}^{-}} w_{s}, \quad V\left(\lambda_{0}, \lambda_{1} ; \phi\right)=\sup _{s \in \mathbf{Z}^{-}} w_{s}
$$

So, by taking into account (4.43), we immediately conclude that all we have to prove is that $\left(w_{n}\right)_{n \in \mathbf{Z}}$ satisfies

$$
\inf _{s \in \mathbf{Z}^{-}} w_{s} \leq w_{n} \leq \sup _{s \in \mathbf{Z}^{-}} w_{s} \quad \forall n \in \mathbf{N} .
$$

We restrict ourselves to show that

$$
w_{n} \geq \inf _{s \in \mathbf{Z}^{-}} w_{s} \quad \text { for every } n \in \mathbf{N} .
$$

In a similar manner, one can prove that

$$
w_{n} \leq \sup _{s \in \mathbf{Z}^{-}} w_{s} \quad \text { for every } n \in \mathbf{N} .
$$

In the rest of the proof we will establish (4.46). To this end, it suffices to show that, for any real number $D$ with $D<\inf _{s \in \mathbf{Z}^{-}} w_{s}$, it holds

$$
w_{n}>D \quad \forall n \in \mathbf{N} .
$$

Let us consider an arbitrary real number $D$ with $D<\inf _{s \in \mathbf{Z}^{-}} w_{s}$. Then we obviously have

$$
w_{n}>D \quad \text { for } n \in \mathbf{Z}^{-} \text {. }
$$

Assume, for the sake of contradiction, that (4.48) fails. Then, because of (4.49), there exists an integer $n_{0}>0$ so that

$$
\begin{gathered}
w_{n}>D \quad \text { for } n \in \mathrm{Z} \text { with } n \leq n_{0}-1, \\
w_{n_{0}} \leq D .
\end{gathered}
$$


Hence, by using the hypothesis that $\left(G_{n}\right)_{n \in \mathbf{N}-\{0\}}$ and $\left(K_{n}\right)_{n \in \mathbf{N}-\{0\}}$ are nonpositive and that $\left(K_{n}\right)_{n \in \mathrm{N}-\{0\}}$ is not eventually identically zero and taking into account the assumption that $\lambda_{0} \leq 1$, from (4.41) we obtain

$$
\begin{aligned}
& D \geq w_{n_{0}}>D\left\{-\sum_{j=1}^{\infty} \lambda_{1}^{-j} G_{j}+\left(1-\frac{1}{\lambda_{0}}\right) \sum_{j=1}^{\infty} \lambda_{0}^{-j} G_{j}\left[\sum_{r=n_{0}-j}^{n_{0}-1}\left(\frac{\lambda_{0}}{\lambda_{1}}\right)^{n_{0}-r}\right]\right. \\
& \left.-\frac{1}{\lambda_{0}} \sum_{j=1}^{\infty} \lambda_{0}^{-j} K_{j}\left[\sum_{r=n_{0}-j}^{n_{0}-1}\left(\frac{\lambda_{0}}{\lambda_{1}}\right)^{n_{0}-r}\right]\right\} \\
& =D\left\{-\sum_{j=1}^{\infty} \lambda_{1}^{-j} G_{j}+\left(1-\frac{1}{\lambda_{0}}\right) \sum_{j=1}^{\infty} \lambda_{0}^{-j} G_{j}\left[\sum_{\nu=1}^{j}\left(\frac{\lambda_{0}}{\lambda_{1}}\right)^{\nu}\right]\right. \\
& \left.-\frac{1}{\lambda_{0}} \sum_{j=1}^{\infty} \lambda_{0}^{-j} K_{j}\left[\sum_{\nu=1}^{j}\left(\frac{\lambda_{0}}{\lambda_{1}}\right)^{\nu}\right]\right\} \\
& =\frac{D}{\lambda_{0}-\lambda_{1}}\left\{-\left(\lambda_{0}-\lambda_{1}\right) \sum_{j=1}^{\infty} \lambda_{1}^{-j} G_{j}+\left(\lambda_{0}-1\right) \sum_{j=1}^{\infty} \lambda_{0}^{-j} G_{j}\left[\left(\frac{\lambda_{0}}{\lambda_{1}}\right)^{j}-1\right]\right. \\
& \left.-\sum_{j=1}^{\infty} \lambda_{0}^{-j} K_{j}\left[\left(\frac{\lambda_{0}}{\lambda_{1}}\right)^{j}-1\right]\right\} \\
& =\frac{D}{\lambda_{0}-\lambda_{1}}\left\{-\left[\left(\lambda_{0}-1\right)-\left(\lambda_{1}-1\right)\right] \sum_{j=1}^{\infty} \lambda_{1}^{-j} G_{j}+\left(\lambda_{0}-1\right) \sum_{j=1}^{\infty}\left(\lambda_{1}^{-j}-\lambda_{0}^{-j}\right) G_{j}\right. \\
& \left.-\sum_{j=1}^{\infty}\left(\lambda_{1}^{-j}-\lambda_{0}^{-j}\right) K_{j}\right\} \\
& =\frac{D}{\lambda_{0}-\lambda_{1}}\left\{\left[-\left(\lambda_{0}-1\right) \sum_{j=1}^{\infty} \lambda_{0}^{-j} G_{j}+\sum_{j=1}^{\infty} \lambda_{0}^{-j} K_{j}\right]\right. \\
& \left.-\left[-\left(\lambda_{1}-1\right) \sum_{j=1}^{\infty} \lambda_{1}^{-j} G_{j}+\sum_{j=1}^{\infty} \lambda_{1}^{-j} K_{j}\right]\right\} \\
& =\frac{D}{\lambda_{0}-\lambda_{1}}\left[\left(\lambda_{0}-1-a\right)-\left(\lambda_{1}-1-a\right)\right]=D \text {. }
\end{aligned}
$$

This contradiction shows that (4.48) holds true.

The proof of the theorem is now complete.

Proof of Theorem 3.9. First, let us notice that the main difference between the neutral case and the non-neutral one is the existence (in the neutral case) of the terms

$$
\sum_{j=1}^{\infty} \lambda_{1}^{-j} G_{j} w_{n-j}, \quad\left(1-\frac{1}{\lambda_{0}}\right) \sum_{j=1}^{\infty} \lambda_{0}^{-j} G_{j}\left[\sum_{r=n-j}^{n-1}\left(\frac{\lambda_{0}}{\lambda_{1}}\right)^{n-r} w_{r}\right]
$$


in (4.41), which do not appear in the non-neutral case. In the special case of the (non-neutral) Volterra difference equation (2.3), (4.41) becomes

$$
w_{n}=-\frac{1}{\lambda_{0}} \sum_{j=1}^{\infty} \lambda_{0}^{-j} K_{j}\left[\sum_{r=n-j}^{n-1}\left(\frac{\lambda_{0}}{\lambda_{1}}\right)^{n-r} w_{r}\right] \quad \text { for } n \in \mathbf{N} \text {. }
$$

The need for assuming, in Theorem 3.7, that the root $\lambda_{0}$ of the characteristic equation (2.8) is such that $\lambda_{0} \leq 1$ is due only to the existence of the second of the above terms in (4.41). After the above observations, we omit the proof of the theorem.

Proof of Proposition 3.11. Assume that there exists another positive root $\lambda_{1}$ of the characteristic equation (2.8) with $\lambda_{1}<\lambda_{0}$ such that (3.17) holds. Clearly,

$$
\sum_{j=1}^{\infty} \lambda_{1}^{-j} G_{j}, \quad \sum_{j=1}^{\infty} \lambda_{1}^{-j} K_{j} \quad \text { exist in } \mathbf{R} .
$$

So, since $\left(G_{n}\right)_{n \in \mathbf{N}-\{0\}}$ and $\left(K_{n}\right)_{n \in \mathbf{N}-\{0\}}$ are nonpositive, we must have

$$
\sum_{j=1}^{\infty} \lambda_{1}^{-j}\left|G_{j}\right|<\infty, \quad \sum_{j=1}^{\infty} \lambda_{1}^{-j}\left|K_{j}\right|<\infty .
$$

(This fact can also be obtained from (3.17).) This guarantees that

$$
\sum_{j=1}^{\infty} \lambda^{-j}\left|G_{j}\right|<\infty, \quad \sum_{j=1}^{\infty} \lambda^{-j}\left|K_{j}\right|<\infty, \quad \forall \lambda \geq \lambda_{1}
$$

and consequently the formula

$$
F(\lambda)=(\lambda-1)\left(1+\sum_{j=1}^{\infty} \lambda^{-j} G_{j}\right)-a-\sum_{j=1}^{\infty} \lambda^{-j} K_{j} \quad \text { for } \lambda \geq \lambda_{1}
$$

defines a real-valued function $F$ on the interval $\left[\lambda_{1}, \infty\right)$. It follows from assumption (3.17) that

$$
\sum_{j=1}^{\infty} \lambda^{-j} j\left|G_{j}\right|<\infty, \quad \sum_{j=1}^{\infty} \lambda^{-j} j\left|K_{j}\right|<\infty, \quad \forall \lambda \geq \lambda_{1},
$$

which ensures that $F$ is differentiable on $\left[\lambda_{1}, \infty\right)$ with

$$
F^{\prime}(\lambda)=1+\sum_{j=1}^{\infty} \lambda^{-j}\left[1-\left(1-\frac{1}{\lambda}\right) j\right] G_{j}+\frac{1}{\lambda} \sum_{j=1}^{\infty} \lambda^{-j} j K_{j} \quad \text { for } \lambda \geq \lambda_{1} .
$$

Furthermore, by using the hypothesis that $\left(G_{n}\right)_{n \in \mathbf{N}-\{0\}}$ and $\left(K_{n}\right)_{n \in \mathbf{N}-\{0\}}$ are nonpositive and $\left(K_{n}\right)_{n \in \mathbf{N}-\{0\}}$ is not eventually identically zero, it is not difficult to check that $F^{\prime}$ is strictly increasing on the interval $\left[\lambda_{1}, 1\right]$. (We notice that $0<\lambda_{1}<\lambda_{0} \leq 1$.)

Now, observe that $F\left(\lambda_{1}\right)=F\left(\lambda_{0}\right)=0$, and so an application of Rolle's theorem ensures the existence of a real number $\xi$ with $\lambda_{1}<\xi<\lambda_{0}$ so that $F^{\prime}(\xi)=0$. Since $F^{\prime}$ is strictly 
increasing on $[\xi, 1]$, it follows that $F^{\prime}$ is always positive on $(\xi, 1]$. Hence, as $\xi<\lambda_{0} \leq 1$, we conclude, in particular, that $F^{\prime}\left(\lambda_{0}\right)>0$, namely that

$$
1+\sum_{j=1}^{\infty} \lambda_{0}^{-j}\left[1-\left(1-\frac{1}{\lambda_{0}}\right) j\right] G_{j}+\frac{1}{\lambda_{0}} \sum_{j=1}^{\infty} \lambda_{0}^{-j} j K_{j}>0 .
$$

By taking into account the fact that $\left(G_{n}\right)_{n \in \mathbf{N}-\{0\}}$ and $\left(K_{n}\right)_{n \in \mathbf{N}-\{0\}}$ are nonpositive and that $\lambda_{0} \leq 1$, we see that the last inequality can equivalently be written as follows

$$
1-\sum_{j=1}^{\infty} \lambda_{0}^{-j}\left(1+\left|1-\frac{1}{\lambda_{0}}\right| j\right)\left|G_{j}\right|-\frac{1}{\lambda_{0}} \sum_{j=1}^{\infty} \lambda_{0}^{-j} j\left|K_{j}\right|>0,
$$

which means that $\lambda_{0}$ has the property (2.10).

The proof of the proposition is complete.

Proof of Proposition 3.12. The proof will be omitted since it is similar to that of Proposition 3.11. We restrict ourselves only to noting that here we have the differentiable real-valued function $F_{0}$ defined by

$$
F_{0}(\lambda)=\lambda-1-a-\sum_{j=1}^{\infty} \lambda^{-j} K_{j} \quad \text { for } \lambda \geq \lambda_{1}
$$

instead of $F$. We note that $F_{0}^{\prime}$ is strictly increasing on the whole interval $\left[\lambda_{1}, \infty\right)$.

Proof of Lemma 3.13. (I) Let us consider the case where $a=0$. Then the characteristic equation (2.8) takes the form

$$
(\lambda-1)\left(1+\sum_{j=1}^{\infty} \lambda^{-j} G_{j}\right)=\sum_{j=1}^{\infty} \lambda^{-j} K_{j}
$$

From the hypothesis that $\left(K_{n}\right)_{n \in \mathrm{N}-\{0\}}$ is nonpositive and not eventually identically zero, it follows that

$$
\sum_{j=1}^{\infty} K_{j}<0
$$

Consequently, $\lambda=1$ cannot be a root of (4.63).

(II) Assume that (4.63) has a positive $\operatorname{root} \mu$ with $\mu>1$. Then

$$
(\mu-1)\left(1+\sum_{j=1}^{\infty} \mu^{-j} G_{j}\right)=\sum_{j=1}^{\infty} \mu^{-j} K_{j}
$$

In view of the fact that $\left(G_{n}\right)_{n \in \mathbf{N}-\{0\}}$ is nonpositive and because of the assumption (3.19), we get

$$
1+\sum_{j=1}^{\infty} \mu^{-j} G_{j} \geq 1+\sum_{j=1}^{\infty} G_{j}=1-\sum_{j=1}^{\infty}\left|G_{j}\right| \geq 0 .
$$


Thus,

$$
(\mu-1)\left(1+\sum_{j=1}^{\infty} \mu^{-j} G_{j}\right) \geq 0
$$

On the other hand, since $\left(K_{n}\right)_{n \in \mathbf{N}-\{0\}}$ is nonpositive and not eventually identically zero, we have

$$
\sum_{j=1}^{\infty} \mu^{-j} K_{j}<0
$$

We have thus arrived at a contradiction.

(III) A particular consequence of assumption (3.21) is that

$$
\sum_{j=1}^{\infty} j\left|K_{j}\right|<\infty
$$

Assumption (3.20) and (4.69) imply, in particular, that

$$
\sum_{j=1}^{\infty}\left|G_{j}\right|<\infty, \quad \sum_{j=1}^{\infty}\left|K_{j}\right|<\infty
$$

(Note that the first of these facts can also be obtained from (3.21).) Thus, we can immediately conclude that

$$
\sum_{j=1}^{\infty} \lambda^{-j}\left|G_{j}\right|<\infty, \quad \sum_{j=1}^{\infty} \lambda^{-j}\left|K_{j}\right|<\infty, \quad \forall \lambda \geq 1 .
$$

So, the formula

$$
F(\lambda)=(\lambda-1)\left(1+\sum_{j=1}^{\infty} \lambda^{-j} G_{j}\right)-a-\sum_{j=1}^{\infty} \lambda^{-j} K_{j} \quad \text { for } \lambda \geq 1
$$

introduces a real-valued function $F$ on the interval $[1, \infty)$. From (3.20) and (4.69), it follows that

$$
\sum_{j=1}^{\infty} \lambda^{-j} j\left|G_{j}\right|<\infty, \quad \sum_{j=1}^{\infty} \lambda^{-j} j\left|K_{j}\right|<\infty, \quad \forall \lambda \geq 1
$$

and consequently the function $F$ is differentiable on $[1, \infty)$ with

$$
F^{\prime}(\lambda)=1+\sum_{j=1}^{\infty} \lambda^{-j}\left[1-\left(1-\frac{1}{\lambda}\right) j\right] G_{j}+\frac{1}{\lambda} \sum_{j=1}^{\infty} \lambda^{-j} j K_{j} \quad \text { for } \lambda \geq 1 .
$$


Furthermore, by the hypothesis that $\left(G_{n}\right)_{n \in \mathbf{N}-\{0\}}$ and $\left(K_{n}\right)_{n \in \mathbf{N}-\{0\}}$ are nonpositive and $\left(K_{n}\right)_{n \in \mathbf{N}-\{0\}}$ is not eventually identically zero, we obtain for $\lambda>1$

$$
\begin{aligned}
F^{\prime}(\lambda) & =1-\sum_{j=1}^{\infty} \lambda^{-j}\left|G_{j}\right|+\left(1-\frac{1}{\lambda}\right) \sum_{j=1}^{\infty} \lambda^{-j} j\left|G_{j}\right|-\frac{1}{\lambda} \sum_{j=1}^{\infty} \lambda^{-j} j\left|K_{j}\right| \\
& >1-\sum_{j=1}^{\infty}\left|G_{j}\right|-\sum_{j=1}^{\infty} j\left|K_{j}\right| .
\end{aligned}
$$

Hence, by assumption (3.21), we find

$$
F^{\prime}(\lambda)>0 \quad \text { for every } \lambda>1
$$

This implies that $F$ is strictly increasing on the interval $(1, \infty)$. Since $\left(K_{n}\right)_{n \in \mathbf{N}-\{0\}}$ is nonpositive, assumption (3.22) means that

$$
F(1) \geq 0
$$

Thus, the characteristic equation $(2.8)$ cannot have roots in the interval $(1, \infty)$.

(IV) Assumption (3.22) means that (4.77) is true. Furthermore, assumption (3.23) guarantees, in particular, that

$$
\sum_{j=1}^{\infty} \gamma^{-j}\left|G_{j}\right|<\infty, \quad \sum_{j=1}^{\infty} \gamma^{-j}\left|K_{j}\right|<\infty
$$

and consequently

$$
\sum_{j=1}^{\infty} \lambda^{-j}\left|G_{j}\right|<\infty, \quad \sum_{j=1}^{\infty} \lambda^{-j}\left|K_{j}\right|<\infty, \quad \forall \lambda \geq \gamma .
$$

So, the formula

$$
F(\lambda)=(\lambda-1)\left(1+\sum_{j=1}^{\infty} \lambda^{-j} G_{j}\right)-a-\sum_{j=1}^{\infty} \lambda^{-j} K_{j} \quad \text { for } \lambda \geq \gamma
$$

defines a real-valued function $F$ on the interval $[\gamma, \infty)$. From assumption (3.23) it follows that

$$
\sum_{j=1}^{\infty} \lambda^{-j} j\left|G_{j}\right|<\infty, \quad \sum_{j=1}^{\infty} \lambda^{-j} j\left|K_{j}\right|<\infty, \quad \forall \lambda \geq \gamma,
$$

which ensures that the function $F$ is differentiable on $[\gamma, \infty)$ with

$$
F^{\prime}(\lambda)=1+\sum_{j=1}^{\infty} \lambda^{-j}\left[1-\left(1-\frac{1}{\lambda}\right) j\right] G_{j}+\frac{1}{\lambda} \sum_{j=1}^{\infty} \lambda^{-j} j K_{j} \quad \text { for } \lambda \geq \gamma
$$


By using the hypothesis that $\left(G_{n}\right)_{n \in \mathbf{N}-\{0\}}$ and $\left(K_{n}\right)_{n \in \mathbf{N}-\{0\}}$ are nonpositive and $\left(K_{n}\right)_{n \in \mathbf{N}-\{0\}}$ is not eventually identically zero, we can easily verify that $F^{\prime}$ is strictly increasing on the interval $[\gamma, 1]$. Consequently,

$$
F \text { is strictly convex on }[\gamma, 1] \text {. }
$$

Furthermore, we take into account the fact that $\left(G_{n}\right)_{n \in \mathbf{N}-\{0\}}$ and $\left(K_{n}\right)_{n \in \mathbf{N}-\{0\}}$ are nonpositive to conclude that assumption (3.24) means that

$$
F(\gamma)>0
$$

while assumption (3.25) means that

$$
F(a+1-\delta)<0
$$

A particular consequence of (4.84) is that $\lambda=\gamma$ is not a root of (2.8). Similarily, (4.85) guarantees, in particular, that $\lambda=a+1-\delta$ is not a root of (2.8). Moreover, from (4.77), (4.83), and (4.85) it follows that, in the interval $(a+1-\delta, 1],(2.8)$ has a unique root. Finally, (4.83), (4.84), and (4.85) ensure that, in the interval $(\gamma, a+1-\delta),(2.8)$ has also a unique root.

The lemma has now been proved.

Proof of Lemma 3.14. (I) and (II) These parts can easily be established using the hypothesis that $\left(K_{n}\right)_{n \in \mathrm{N}-\{0\}}$ is nonpositive and not eventually identically zero.

(III) By using the assumption (3.26) and following some arguments similar to those in the proof of Lemma 3.13, we can see that the formula

$$
F_{0}(\lambda)=\lambda-1-a-\sum_{j=1}^{\infty} \lambda^{-j} K_{j} \quad \text { for } \lambda \geq \gamma
$$

defines a real-valued function $F_{0}$, which is differentiable on $[\gamma, \infty)$. Furthermore, the hypothesis that $\left(K_{n}\right)_{n \in \mathrm{N}-\{0\}}$ is nonpositive and not eventually identically zero ensures that $F_{0}^{\prime}$ is strictly increasing on the interval $[\gamma, \infty)$. So,

$$
F_{0} \text { is strictly convex on }[\gamma, \infty) \text {. }
$$

Now, as $\left(K_{n}\right)_{n \in \mathbf{N}-\{0\}}$ is nonpositive, assumption (3.27) means that

$$
F_{0}(\gamma)>0
$$

while assumption (3.28) means that

$$
F_{0}(a+1-\delta)<0
$$

From (4.88) it follows, in particular, that $\lambda=\gamma$ is not a root of (2.9), while (4.89) ensures, in particular, that $\lambda=a+1-\delta$ is not a root of (2.9). Next, by taking into account the fact that $\left(K_{n}\right)_{n \in \mathbf{N}-\{0\}}$ is nonpositive and not eventually identically zero, we see that

$$
F_{0}(a+1)>0 \text {. }
$$


Because of (4.87), (4.89), and (4.90), we conclude that, in the interval $(a+1-\delta, a+1)$, (2.9) has a unique root. Moreover, (4.87), (4.88), and (4.89) guarantee that, in the interval $(\gamma, a+1-\delta),(2.9)$ admits also a unique root.

We have thus completed the proof of our lemma.

\section{Acknowledgment}

The authors are grateful to Prof. Leonid Shaikhet for his useful comments which improved the original manuscript.

\section{References}

[1] R. P. Agarwal, Difference Equations and Inequalities. Theory, Methods, and Applications, Monographs and Textbooks in Pure and Applied Mathematics, vol. 155, Marcel Dekker, New York, 1992.

[2] N. G. de Bruijn, On some linear functional equations, Publicationes Mathematicae Debrecen 1 (1950), 129-134.

[3] R. D. Driver, Some harmless delays, Delay and Functional Differential Equations and Their Applications (Proc. Conf., Park City, Utah, 1972), Academic Press, New York, 1972, pp. 103-119.

[4] R. D. Driver, G. Ladas, and P. N. Vlahos, Asymptotic behavior of a linear delay difference equation, Proceedings of the American Mathematical Society 115 (1992), no. 1, 105-112.

[5] R. D. Driver, D. W. Sasser, and M. L. Slater, The equation $x^{\prime}(t)=a x(t)+b x(t-\tau)$ with "small" delay, The American Mathematical Monthly 80 (1973), 990-995.

[6] S. Elaydi, Stability of Volterra difference equations of convolution type, Proceedings of the Special Program on Dynamical Systems and Ordinary Differential Equations held at the Nankai Institute of Mathematics (Tianjin, 1990/1991) (S. T. Liao, Y. Q. Ye, and T. R. Ding, eds.), Nankai Ser. Pure Appl. Math. Theoret. Phys., vol. 4, World Scientific, New Jersey, 1993, pp. 66-72.

[7] __ An Introduction to Difference Equations, Undergraduate Texts in Mathematics, Springer, New York, 1996.

[8] _ Global stability of difference equations, World Congress of Nonlinear Analysts '92, Vol. I-IV (Tampa, Fla, 1992), de Gruyter, Berlin, 1996, pp. 1131-1138.

[9] S. Elaydi and S. Murakami, Asymptotic stability versus exponential stability in linear Volterra difference equations of convolution type, Journal of Difference Equations and Applications 2 (1996), no. 4, 401-410.

[10] M. V. S. Frasson and S. M. Verduyn Lunel, Large time behaviour of linear functional differential equations, Integral Equations and Operator Theory 47 (2003), no. 1, 91-121.

[11] J. R. Graef and C. Qian, Asymptotic behavior of forced delay equations with periodic coefficients, Communications in Applied Analysis. An International Journal for Theory and Applications 2 (1998), no. 4, 551-564.

[12] I. Györi, Sharp conditions for existence of nontrivial invariant cones of nonnegative initial values of difference equations, Applied Mathematics and Computation 36 (1990), no. 2, part II, 89-111.

[13] J. Jaroš and I. P. Stavroulakis, Necessary and sufficient conditions for oscillations of difference equations with several delays, Utilitas Mathematica. An International Journal of Discrete and Combinatorial Mathematics, and Statistical Design 45 (1994), 187-195.

[14] W. G. Kelley and A. C. Peterson, Difference Equations. An Introduction with Applications, Academic Press, Massachusetts, 1991.

[15] Th. Kiventidis, Positive solutions of integrodifferential and difference equations with unbounded delay, Glasgow Mathematical Journal 35 (1993), no. 1, 105-113. 
[16] I.-G. E. Kordonis, N. T. Niyianni, and Ch. G. Philos, On the behavior of the solutions of scalar first order linear autonomous neutral delay differential equations, Archiv der Mathematik 71 (1998), no. 6, 454-464.

[17] I.-G. E. Kordonis and Ch. G. Philos, On the behavior of the solutions for linear autonomous neutral delay difference equations, Journal of Difference Equations and Applications 5 (1999), no. 3, 219233.

[18] _ Positive solutions of certain difference equations, Differential Equations and Dynamical Systems. An International Journal for Theory and Applications 7 (1999), no. 3, 281-303.

[19] - The behavior of solutions of linear integro-differential equations with unbounded delay, Computers \& Mathematics with Applications. An International Journal 38 (1999), no. 2, 45-50.

[20] I.-G. E. Kordonis, Ch. G. Philos, and I. K. Purnaras, Some results on the behavior of the solutions of a linear delay difference equation with periodic coefficients, Applicable Analysis. An International Journal 69 (1998), no. 1-2, 83-104.

[21] _ On the behavior of solutions of linear neutral integrodifferential equations with unbounded delay, Georgian Mathematical Journal 11 (2004), no. 2, 337-348.

[22] G. Ladas, Ch. G. Philos, and Y. G. Sficas, Existence of positive solutions for certain difference equations, Utilitas Mathematica. An International Journal of Discrete and Combinatorial Mathematics, and Statistical Design 38 (1990), 119-128.

[23] V. Lakshmikantham and D. Trigiante, Theory of Difference Equations. Numerical Methods and Applications, Mathematics in Science and Engineering, vol. 181, Academic Press, Massachusetts, 1988.

[24] R. E. Mickens, Difference Equations. Theory and Applications, 2nd ed., Van Nostrand Reinhold, New York, 1990.

[25] M. J. Norris, Unpublished notes on the delay differential equation $x^{\prime}(t)=b x(t-1)$ where $-1 / e \leqslant$ $b<0$, October 1967.

[26] Ch. G. Philos, Asymptotic behaviour, nonoscillation and stability in periodic first-order linear delay differential equations, Proceedings of the Royal Society of Edinburgh. Section A. Mathematics 128 (1998), no. 6, 1371-1387.

[27] _ On the existence of positive solutions for certain difference equations and inequalities, Journal of Inequalities and Applications 2 (1998), no. 1, 57-69.

[28] Ch. G. Philos and I. K. Purnaras, Periodic first order linear neutral delay differential equations, Applied Mathematics and Computation 117 (2001), no. 2-3, 203-222.

[29] _ An asymptotic result for some delay difference equations with continuous variable, Advances in Difference Equations 2004 (2004), no. 1, 1-10.

[30] _ Asymptotic properties, nonoscillation, and stability for scalar first order linear autonomous neutral delay differential equations, Electronic Journal of Differential Equations 2004 (2004), no. $03,1-17$.

[31] On the behavior of the solutions for certain linear autonomous difference equations, Journal of Difference Equations and Applications 10 (2004), no. 12, 1049-1067.

[32] _ The behavior of solutions of linear Volterra difference equations with infinite delay, Computers \& Mathematics with Applications. An International Journal 47 (2004), no. 10-11, 15551563.

[33] More on the behavior of solutions to linear integrodifferential equations with unbounded delay, Fako de l'Funkcialaj Ekvacioj Japana Matematika Societo. Funkcialaj Ekvacioj. 48 (2005), no. 3, 393-414.

[34] _ The behavior of the solutions of periodic linear neutral delay difference equations, Journal of Computational and Applied Mathematics 175 (2005), no. 2, 209-230.

[35] _ A result on the behavior of the solutions for scalar first order linear autonomous neutral delay differential equations, to appear in Mathematical Proceedings of the Cambridge Philosophical Society. 


\section{Volterra difference equations with infinite delay}

[36] On the behavior of the solutions for certain first order linear autonomous functional differential equations, to appear in Rocky Mountain J. Math.

[37] M. Pituk, The limits of the solutions of a nonautonomous linear delay difference equation, Computers \& Mathematics with Applications. An International Journal 42 (2001), no. 3-5, 543-550.

[38] __ Asymptotic behavior of a nonhomogeneous linear recurrence system, Journal of Mathematical Analysis and Applications 267 (2002), no. 2, 626-642.

[39] A. N. Sharkovsky, Yu. L. Maĭstrenko, and E. Yu. Romanenko, Difference Equations and Their Applications, Mathematics and Its Applications, vol. 250, Kluwer Academic, Dordrecht, 1993.

Ch. G. Philos: Department of Mathematics, University of Ioannina,

P.O. Box 1186, 45110 Ioannina, Greece

E-mail address: cphilos@cc.uoi.gr

I. K. Purnaras: Department of Mathematics, University of Ioannina,

P.O. Box 1186, 45110 Ioannina, Greece

E-mail address: ipurnara@cc.uoi.gr 